\title{
Numerical infinities applied for studying Riemann series theorem and Ramanujan summation
}

\author{
Yaroslav D. Sergeyev \\ University of Calabria, Via Pietro Bucci 42C, 87036 Rende (CS), Italy and \\ the Institute of High Performance Computing and Networking of the National Research Council of Italy and \\ Lobachevsky State University, Nizhni Novgorod, Russia
}

\begin{abstract}
A computational methodology called Grossone Infinity Computing introduced with the intention to allow one to work with infinities and infinitesimals numerically has been applied recently to a number of problems in numerical mathematics (optimization, numerical differentiation, numerical algorithms for solving ODEs, etc.). The possibility to use a specially developed computational device called the Infinity Computer (patented in USA and EU) for working with infinite and infinitesimal numbers numerically gives an additional advantage to this approach in comparison with traditional methodologies studying infinities and infinitesimals only symbolically. The grossone methodology uses the Euclid's Common Notion no. 5 'The whole is greater than the part' and applies it to finite, infinite, and infinitesimal quantities and to finite and infinite sets and processes. It does not contradict Cantor's and non-standard analysis views on infinity and can be considered as an applied development of their ideas. In this paper we consider infinite series and a particular attention is dedicated to divergent series with alternate signs. The Riemann series theorem states that conditionally convergent series can be rearranged in such a way that they either diverge or converge to an arbitrary real number. It is shown here that Riemann's result is a consequence of the fact that symbol $\infty$ used traditionally does not allow us to express quantitatively the number of addends in the series, in other words, it just shows that the number of summands is infinite and does not allows us to count them. The usage of the grossone methodology allows us to see that (as it happens in the case where the number of addends is finite) rearrangements do not change the result for any sum with a fixed infinite number of summands. There are considered some traditional summation techniques such as Ramanujan summation producing results where to divergent series containing infinitely many positive integers negative results are assigned. It is shown that the careful counting of the number of addends in infinite series allows us to avoid this kind of results if grossone-based numerals are used.
\end{abstract}

Keywords: Numerical infinities and infinitesimals, grossone, divergent series, Riemann series theorem, Ramanujan summation. PACS: $02.30 . \mathrm{Lt}, 02.70 .-\mathrm{c}, 02.10 . \mathrm{De}$

\section{INTRODUCTION}

Infinite and infinitesimal quantities continue to attract a great attention of mathematicians and computer scientists (see $[1,2,3,4,5,6,7,8,9,10])$ and references given therein). Traditionally, theories proposed to work with these quantities are symbolical and they do not allow one to execute numerical computations on a computer. A recent computational approach developed in $[11,12,13,14]$ proposes numerical infinities and infinitesimals and offers new attractive possibilities in computations. This methodology uses a numeral system that avoids situations of the type $\infty+1=\infty$ providing results ensuring that if $a$ is a numeral written in this system, then for any $a$ (i.e., $a$ can be finite, infinite, or infinitesimal) it follows $a+1>a$. Even though it works with infinity in accordance with Euclid's Common Notion no. 5 'The whole is greater than the part' it does not contradict traditional approaches. An infinite unit of measure expressed by the numeral (1) called grossone is introduced as the number of elements of the set, $\mathbb{N}$, of natural numbers. Grossone is introduced by describing its properties postulated by the Infinite Unit Axiom (see [12, 13, 14]) added to axioms for real numbers. The numeral (1) allows one to construct different numerals expressing different infinite and infinitesimal numbers and to execute computations with all of them. As a result, instead of the usual symbol $\infty$ different infinite (and infinitesimal) numerals can be used in numerical computations. Indeterminate forms are not present and, for example, the following relations hold for infinite numbers (1), (1) ${ }^{2}$ and infinitesimals (1) ${ }^{-1}$, (1) ${ }^{-2}$ as for any other (finite, infinite, or infinitesimal) number expressible in the (1)-based numeral system:

$$
\begin{aligned}
& 0 \cdot(1)=(1) \cdot 0=0, \quad(1)-(1)=0, \quad \frac{1}{(1)}=1, \quad \frac{1^{-1}}{(1)^{-1}}=1, \quad 1^{0}=1, \quad 1^{\circledR}=1, \quad 0^{\circledR}=0, \quad 0 \cdot(1)^{-1}=(1)^{-1} \cdot 0=0, \\
& \left.(1)^{-1}>0, \quad(1)^{-2}>0, \quad(1)^{-1}-(1)^{-1}=0, \quad(1)^{-2}-(1)^{-2}=0, \quad(1)^{-1}\right)^{0}=1, \quad(1) \cdot(1)^{-1}=1, \quad(1) \cdot(1)^{-2}=(1)^{-1} \text {. }
\end{aligned}
$$


A computational device called the Infinity Computer (patented in USA and EU, see [15]) working numerically with finite numbers and (1)-based infinities and infinitesimals in a unique computational framework has been introduced recently. A number of papers studying connections of the (1)-based approach to the historical panorama of ideas dealing with infinities and infinitesimals (see $[16,17,18,19,20])$ have been published. In particular, metamathematical investigations on the new theory and its non-contradictory can be found in [17]. The (1)-based methodology has been successfully applied in several areas of Mathematics and Computer Science: single and multiple criteria optimization (see [21, 22, 23, 24, 25]), cellular automata (see [26, 27]), Euclidean and hyperbolic geometry (see [28, 29]), percolation (see [30]), fractals (see [31, 32, 33, 34, 35]), infinite series and the Riemann zeta function (see [36, 37, 38, $39,40]$ ), the first Hilbert problem, Turing machines, and supertasks (see [41, 42, 20, 43]), numerical differentiation and numerical solution of ordinary differential equations (see [44, 45, 46, 47, 48]), etc. In this paper, divergent series and Ramanujan summation are studied.

\section{From divergent series to sums with a fixed infinite number of addends}

Suppose that we consider two divergent series

$$
S_{1}=\sum_{i=1}^{\infty} a_{i}, \quad S_{2}=\sum_{i=1}^{\infty} b_{i} .
$$

The traditional analysis can say a little about them and does not allow us to execute numerical computations with them, e.g., such operations as $S_{2} / S_{1}$ and $S_{2}-S_{1}$ are not defined.

Since the (1)-based methodology allows us to express not only different finite numbers but also different infinite numbers, such records as $S_{1}=a_{1}+a_{2}+\ldots$ or $\sum_{i=1}^{\infty} a_{i}$ become unprecise and it is required to indicate explicitly infinite numbers, $k$ and $n$, being the number of summands in the respective sums $S_{1}$ and $S_{2}$ from (1). Thus, we get more precise definitions of these sums where the infinite numbers of addends are specified

$$
S_{1}(k)=a_{1}+a_{2}+\ldots+a_{k-1}+a_{k}, \quad S_{2}(n)=b_{1}+b_{2}+\ldots+b_{k-1}+b_{n} .
$$

Notice that this specification is exactly the same as is required in the finite case. In fact, we know that when we have a sum with a finite number of addends it is not sufficient to say that the number, $l$, of addends in the sum is finite, it is necessary to assign explicitly a value to $l$ applying for this purpose numerals available in a traditional numeral system chosen to express finite numbers. For different finite values of $l$ the result of summation can be different.

It is necessary to stress that (1) and (2) describe the same mathematical objects - sums with an infinite number of addends - but with different accuracies. In the case (1), the numeral $\infty$ is used and the number of addends is not fixed - it is just said that it is infinite. The (1)-based numerals allow us to observe that each divergent series is not a unique object with a generic infinite number of addends expressed by the numeral $\infty$. Records from (2) were visible like unique objects due to the low accuracy of the numeral $\infty$. Now we are able to distinguish different sums $S_{1}(k)$ and $S_{2}(n)$ and for different infinite values of $k$ and $n$ different results can be obtained in (2).

Notice also that it would not be correct to say that, e.g., the record $S_{1}=\sum_{i=1}^{\infty} a_{i}$ from (1) represents a 'true infinite series that includes all possible addends $a_{i}$ ' whereas records (2) where the number of summands should be expressed by concrete infinite (1)-based numbers are some 'partial infinite sums'. The sentence 'all possible addends' means that the numeral $\infty$ being the upper index of summation represents the largest possible integer (if it is not the largest one then we do not consider all possible addends). Thus, the operation $\infty+1$ should be prohibited since we are not allowed to increase $\infty$ that represents the largest possible integer (notice that an interesting arithmetic where there exists the largest integer $K$ and the operation $K+1$ is not allowed is given in [19]). Analogously, the operation $\infty-1$ should give a result different from $\infty$ since in this case we have less addends in the sum. In other words, the assumption that $S_{1}=\sum_{i=1}^{\infty} a_{i}$ includes all possible addends $a_{i}$ leads to the necessity to attribute to the symbol $\infty$ properties that are different from its traditional usage, where relations $\infty+1=\infty$ and $\infty-1=\infty$, etc. hold. Therefore, the meaning 'sum of all possible addends $a_{i}$ ' cannot be attributed to the record $S_{1}=\sum_{i=1}^{\infty} a_{i}$ if the numeral $\infty$ is used in its conventional sense. Thus, records (1) and (2) describe the same mathematical objects - sums with an infinite number of addends but with different accuracies.

\section{Riemann series theorem and Ramanujan summation}

Let us consider now series with alternating signs. Traditionally, convergence of series is studied and only for unconditionally convergent series rearrangements of addends give the same result as for the original series before 
the rearrangement. The Riemann series theorem states that conditionally convergent series can be rearranged in such a way that they either diverge or converge to an arbitrary real number. In its turn, the (1)-based methodology shows that Riemann's result is a consequence of the weak numeral system used traditionally to work with infinite series. In fact, the usage of (1) allows us to see that (as it happens in the case where the number of addends is finite) rearrangements do not change the result for any sum with a fixed (finite or infinite) number of summands.

Let us start our consideration from the famous series $S_{3}=1-1+1-1+1-1+\ldots$ In the literature there exist many approaches giving different answers regarding the value of this series (see, e.g., [49]). All of them use various notions of average (for instance, Cesàro summation assigns the value 0.5 to $S_{3}$ ). However, sum and its average of any kind are different notions. In our approach we do not appeal to any kind of average and calculate the required sum directly. To do this, we should indicate explicitly the number of items, $k$, in the sum. Then it follows that

$$
S_{3}(k)=\underbrace{1-1+1-1+1-1+1-\ldots}_{k \text { addends }}= \begin{cases}0, & \text { if } k=2 n, \\ 1, & \text { if } k=2 n+1,\end{cases}
$$

and it is not important whether $k$ is finite or infinite. For example, $S_{3}(1)=0$ since (1) is even (see [13]). Analogously, $\left.S_{3}(1)-1\right)=1$ because $11-1$ is odd.

As it happens in the cases where the number of addends in a sum is finite, the result of summation does not depend on the way the summands are rearranged. In fact, if we know the exact infinite number of addends and the order the signs are alternated is clearly defined, we know also the exact number of positive and negative addends in the sum. Let us illustrate this point by supposing, for instance, that we want to rearrange addends in the sum $S_{3}(21)$ as follows

$$
S_{3}(21)=1+1-1+1+1-1+1+1-1+\ldots
$$

Traditional mathematical tools used to study divergent series give an impression that this rearrangement modifies the result. However, in the (1)-based framework we know that this is just a consequence of the weak lens used to observe infinite numbers. In fact, thanks to (1) we are able to fix an infinite number of summands. In our example the sum has 21 addends, the number 21 is even and, therefore, it follows from (3) that $S_{3}(21)=0$. This means also that in the sum there are (1) positive and (1) negative items. As a result, addition of the groups $1+1-1$ considered above can continue only until the positive units present in the sum will not finish and then there will be necessary to continue to add only negative summands. More precisely, we have

$$
S_{3}(2(1)=\underbrace{1+1-1+1+1-1+\ldots+1+1-1}_{(\mathbb{\infty}) \text { positive and } \frac{\mathbb{1}}{2} \text { negative addends }} \underbrace{-1-1-\ldots-1-1-1}_{\frac{\mathbb{\Phi}}{2} \text { negative addends }}=0,
$$

where the result of the first part in this rearrangement is calculated as $(1+1-1) \cdot \frac{\Phi}{2}=\frac{\Phi}{2}$ and the result of the second part summing up negative units is equal to $-\frac{\mathbb{1}}{2}$ giving so the same final result as (3). It becomes clear from (4) the origin of the Riemann series theorem. In fact, the second part of (4) containing only negative units is invisible if one works with the traditional numeral $\infty$.

Let us consider now the following divergent series $S_{4}=1-2+3-4+\ldots$ It can be considered as difference of two arithmetic progressions (see [37]) after we have fixed the infinite number of items, $k$, in the sum $S_{4}(k)$. Suppose that $k=$ (1). Then it follows

$$
\begin{gathered}
\left.S_{4}(1)=1-2+3-4+\ldots-(1)-2\right)+(1-1)-1= \\
(1+3+5+\ldots+(1-3)+(1-1))-(2+4+6+\ldots+(1-2)+(1)= \\
0.25\left(1+(1-1)(1)-0.25(2+\text { (1) })(1)=0.25(1)^{2}-21-(1)^{2}\right)=-0.51 .
\end{gathered}
$$

Notice that some traditional summation techniques can be interpreted as certain weighted averages on sums having (1) addends (see [40,50] for a detailed discussion). Let us see now what happens in the (1)-based framework in situations where certain summation techniques produce results where to divergent series containing infinitely many positive integers negative results are assigned. In particular, we consider the famous result of Ramanujan

$$
c=1+2+3+4+5+\ldots=-1 / 12
$$

viewed through the lens of the (1) methodology. To do this let us first remind how Ramanujan derives (6). He multiplies the left-hand part of (6) by 4 and then subtracts the result from (6) as follows

$$
\begin{aligned}
& c=1+2+3+4+5+6+\ldots \\
& 4 c=4+8+12+\ldots \\
& -3 c=\begin{array}{llllll}
1 & -2 & +3 & -4 & +5 & -6+\ldots
\end{array}
\end{aligned}
$$


Ramanujan then uses the result (considered in various forms by Euler, Cesàro and Hölder) attributing to the alternating series $1-2+3-4+\ldots$ the value $\frac{1}{4}$ as the formal power series expansion of the function $\frac{1}{(1+x)^{2}}$ for $x=1$, i.e.,

$$
1-2+3-4+\ldots=1 / 4 \text {. }
$$

Thus, it follows from (7) and (8) that

$$
-3 c=1-2+3-4+5-6+\ldots=1 / 4,
$$

from where Ramanujan gets (6).

Let us use now the (1) lens and observe Ramanujan's procedure through it. First, it is necessary to indicate an infinite number of addends, $n$, in the sum

$$
c(n)=1+2+3+4+5+\ldots+n .
$$

The (1) methodology allows us to compute this sum for infinite values of $n$ directly (see [37]) and to show that for infinite (and finite) values of $n$ it follows

$$
c(n)=0.5 n(1+n)
$$

and for $n=$ (1) we have 0.5(1) $(1+$ (1) ) that is sum of natural numbers (see [37] for a detailed discussion).

Let us now return to Ramanujan summation and consider the main trick of (7) consisting of displacement of addends in its second line. Without loss of generality let us see what happens with this shift in the case we have $n=(1)$ addends in $c(n)$. This means that sum (9) has (1) summands. However, the displacement of (7) proposes to sum up each number $i$ from the first line of (7) with the number $4 \cdot \frac{i}{2}$ for all even $i$ and odd $i$ are summed up with zeros, i.e., they are just moved directly to the third line of (7). The important novelty offered by the (1) methodology is that now we know that since $n=$ (1) in our case we have $1 \leq i \leq$ (1). This means that to 0.51 even addends in (9) there will be added the first 0.51 numbers from (10), the last addend (1) from (9) corresponds to the number $4 \cdot \frac{1}{2}$ in (10). As a consequence, in (10) there will remain 0.5 (1) more addends, namely, they are

$$
4 \cdot\left(\frac{1}{2}+1\right)+4 \cdot\left(\frac{1}{2}+2\right)+\ldots+4 \cdot(1)
$$

The record (7) does not allow us to observe them whereas thanks to (1) these summands are perfectly visible and, therefore, (7) can be re-written using (9) - (11) in the following, more accurate than it is done traditionally, way

$$
\begin{array}{rccc}
c(1) & = & 1+2+3+4+5+\ldots+1)-1+(1) \\
4 c(1) & = & 4+8+\ldots & +4 \frac{1}{2}+4\left(\frac{1}{2}+1\right)+\ldots+41 \\
-3 c(1) & = & 1-2+3-4+5+\ldots+(1)-1-(1)-4\left(\frac{1}{2}+1\right)-\ldots-41
\end{array}
$$

Let us compute now the right-hand part of the third line of (12) by using (5) for the first (1) addends and the arithmetical progression formula for the remaining 0.51 summands

$$
\begin{gathered}
1-2+3-4+5+\ldots+1)-1-1)-4\left(\frac{1}{2}+1\right)-4\left(\frac{1}{2}+2\right)-\ldots-41= \\
\left.-\frac{1}{2}-4\left(\left(\frac{1}{2}+1\right)+\left(\frac{1}{2}+2\right)+\ldots+(1)\right)=-\frac{1}{2}-4 \cdot \frac{1}{2}\left(\left(\frac{1}{2}+1\right)+(1)\right)=-3 \frac{1}{2}(1)+1\right) .
\end{gathered}
$$

As was expected, the obtained result shows that the third line of (12) is consistent with (10) for $n=(1$.

Thus, it has been shown in this paper that Riemann's result on rearrangements of addends in series is a consequence of the fact that symbol $\infty$ used traditionally does not allow us to express quantitatively the infinite number of addends in the series. The usage of the grossone methodology allows us to see that (as it happens in the case where the number of addends is finite) rearrangements of addends do not change the result for any sum with a fixed infinite number of summands. This happens because if one knows the number of addends and the rule used to alternate their signs, he/she knows the number of positive and negative addends. Ramanujan summation producing results where to divergent series containing infinitely many positive integers negative results are assigned was considered here. It has been shown that the careful counting of the number of addends in infinite series allows us to avoid this kind of results if (1)-based numerals are used. 


\section{REFERENCES}

1. J. Bagaria, and M. Magidor, Transactions of the American Mathematical Society 366, 1857-1877 (2014).

2. G. Cantor, Contributions to the founding of the theory of transfinite numbers, Dover Publications, New York, 1955.

3. J. Conway, and R. Guy, The Book of Numbers, Springer-Verlag, New York, 1996.

4. G. Hardy, Orders of infinity, Cambridge University Press, Cambridge, 1910.

5. M. Heller, and W. H. Woodin, editors, Infinity: New Research Frontiers, Cambridge University Press, Cambridge, 2011.

6. A. Kanamori, The Higher Infinite: Large cardinals in set theory from their beginnings, Springer, Berlin Heidelberg, 2003.

7. G. Leibniz, and J. Child, The Early Mathematical Manuscripts of Leibniz, Dover Publications, New York, 2005.

8. I. Newton, Method of Fluxions, 1671.

9. A. Robinson, Non-standard Analysis, Princeton Univ. Press, Princeton, 1996.

10. J. Wallis, Arithmetica infinitorum, 1656.

11. Y. D. Sergeyev, Arithmetic of Infinity, Edizioni Orizzonti Meridionali, CS, 2003, 2nd ed. 2013.

12. Y. D. Sergeyev, Informatica 19(4), 567-596 (2008).

13. Y. D. Sergeyev, Rendiconti del Seminario Matematico dell'Università e del Politecnico di Torino 68(2), 95-113 (2010).

14. Y. D. Sergeyev, "Numerical computations with infinite and infinitesimal numbers: Theory and applications," in Dynamics of Information Systems: Algorithmic Approaches, edited by S. A., and P. P.M., Springer, New York, 2013, pp. 1-66.

15. Y. D. Sergeyev, Computer system for storing infinite, infinitesimal, and finite quantities and executing arithmetical operations with them, USA patent 7,860,914, 2010.

16. G. Lolli, Applied Mathematics and Computation 218(16), 7979-7988 (2012).

17. G. Lolli, Applied Mathematics and Computation 255, 3-14 (2015).

18. M. Margenstern, p-Adic Numbers, Ultrametric Analysis and Applications 3(3), 196-204 (2011).

19. F. Montagna, G. Simi, and A. Sorbi, Communications in Nonlinear Science and Numerical Simulation 21(1-3), 52-69 (2015).

20. Y. D. Sergeyev, and A. Garro, Informatica 21(3), 425-454 (2010).

21. M. Cococcioni, M. Pappalardo, and Y. D. Sergeyev, Applied Mathematics and Computation (in press).

22. S. De Cosmis, and R. De Leone, Applied Mathematics and Computation 218(16), 8029-8038 (2012).

23. R. De Leone, Applied Mathematics and Computation (published online 15 May 2017).

24. M. Gaudioso, G. Giallombardo, and M. Mukhametzhanov, Applied Mathematics and Computation (in press).

25. A. Žilinskas, Applied Mathematics and Computation 218(16), 8131-8136 (2012).

26. L. D'Alotto, Indian Journal of Mathematics 55, 143-158 (2013).

27. L. D'Alotto, Applied Mathematics and Computation 255, 15-24 (2015).

28. M. Margenstern, Applied Mathematics and Computation 218(16), 8005-8018 (2012).

29. M. Margenstern, Communications in Nonlinear Science and Numerical Simulation 21(1-3), 3-11 (2015).

30. D. Iudin, Y. D. Sergeyev, and M. Hayakawa, Applied Mathematics and Computation 218(16), 8099-8111 (2012).

31. F. Caldarola, Applied Mathematics and Computation (in press).

32. Y. D. Sergeyev, Chaos, Solitons \& Fractals 33, 50-75 (2007).

33. Y. D. Sergeyev, Chaos, Solitons \& Fractals 42(5), 3042-3046 (2009).

34. Y. D. Sergeyev, Informatica 22(4), 559-576 (2011).

35. Y. D. Sergeyev, Communications in Nonlinear Science and Numerical Simulation 31(1-3), 21-29 (2016).

36. Y. D. Sergeyev, Nonlinear Analysis Series A: Theory, Methods \& Applications 71(12), e1688-e1707 (2009).

37. Y. D. Sergeyev, p-Adic Numbers, Ultrametric Analysis and Applications 3(2), 129-148 (2011).

38. Y. D. Sergeyev, "Computations with grossone-based infinities," in Unconventional Computation and Natural Computation: Proc. of the 14th International Conference UCNC 2015, edited by C. C.S., and D. M.J., Springer, New York, 2015, vol. LNCS 9252, pp. 89-106.

39. Y. D. Sergeyev, Matematica nella Società e nella Cultura: Rivista della Unione Matematica Italiana 8(1), 111-147 (2015).

40. A. Zhigljavsky, Applied Mathematics and Computation 218(16), 8064-8076 (2012).

41. D. Rizza, "Supertasks and numeral systems," in Proc. of the 2nd Intern. Conf. "Numerical Computations: Theory and Algorithms", edited by S. Y. D., K. D.E., D. F., and M. M.S., AIP Publishing, New York, 2016, vol. 1776, p. 090005.

42. Y. D. Sergeyev, Nonlinear Analysis Series A: Theory, Methods \& Applications 72(3-4), 1701-1708 (2010).

43. Y. D. Sergeyev, and A. Garro, Journal of Supercomputing 65(2), 645-663 (2013).

44. P. Amodio, F. Iavernaro, F. Mazzia, M. Mukhametzhanov, and Y. D. Sergeyev, Mathematics and Computers in Simulation (2017), in press.

45. F. Mazzia, Y. D. Sergeyev, F. Iavernaro, P. Amodio, and M. Mukhametzhanov, "Numerical methods for solving ODEs on the Infinity Computer," in Proc. of the 2nd Intern. Conf. "Numerical Computations: Theory and Algorithms", edited by S. Y. D., K. D.E., D. F., and M. M.S., AIP Publishing, New York, 2016, vol. 1776, p. 090033.

46. Y. D. Sergeyev, Optimization Letters 5(4), 575-585 (2011).

47. Y. D. Sergeyev, Applied Mathematics and Computation 219(22), 10668-10681 (2013).

48. Y. D. Sergeyev, M. Mukhametzhanov, F. Mazzia, F. Iavernaro, and P. Amodio, International Journal of Unconventional Computing 12(1), 3-23 (2016).

49. K. Knopp, Theory and Application of Infinite Series, Dover Publications, New York, 1990.

50. A. Zhigljavsky, and V. Kornikov, "Classical areas of mathematics where the concept of grossone could be useful," in Proc. of the 2nd Intern. Conf. "Numerical Computations: Theory and Algorithms", edited by S. Y. D., K. D.E., D. F., and M. M.S., AIP Publishing, New York, 2016, vol. 1776, p. 020004. 